\section{Case Report}

Korean J Transplant 2021;35:124-129 https://doi.org/10.4285/kjt.20.0055

\section{$\mathrm{KJT}$ \\ KOREAN JOURNAL OF TRANSPLANTATION}

\title{
Recipient liver splitting to facilitate piggyback hepatectomy in adult living donor liver transplantation
}

\author{
Sung-Min Kim, Shin Hwang, Deok-Bog Moon, Dong-Hwan Jung \\ Division of Hepatobiliary Surgery and Liver Transplantation, Department of Surgery, Asan Medical Center, \\ University of Ulsan College of Medicine, Seoul, Korea
}

Received December 10, 2020

Revised December 31, 2020

Accepted January 22, 2021

Corresponding author: Shin Hwang Department of Surgery, Asan Medical Center, University of Ulsan College of Medicine, 88 Olympic-ro 43-gil, Songpagu, Seoul 05505, Korea

Tel: +82-2-3010-3930

Fax: +82-2-3010-6701

E-mail: shwang@amc.seoul.kr

\footnotetext{
(c) The Korean Society for Transplantation
This is an Open Access article distributed

(c) The Korean Society for Transplantation
This is an Open Access article distributed under the terms of the Creative Commons Attribution Non-Commercial License (http://creativecommons.org/licenses/ by-nc/4.0/) which permits unrestricted non-commercial use, distribution, and reproduction in any medium, provided the original work is properly cited.
}

\begin{abstract}
Recipient hepatectomy for an enlarged stony-hard liver is a demanding procedure, thus it is often accompanied by massive blood loss. Recipient liver splitting under prolonged hepatic inflow occlusion would facilitate the piggyback recipient hepatectomy. We herein present a case of recipient liver splitting, which was used for living donor liver transplantation (LDLT). A 48-year-old male patient diagnosed with acute-on-chronic liver failure underwent LDLT. During the recipient operation, the native liver was stony-hard and heavily adherent to the retrohepatic inferior vena cava (IVC). During liver mobilization, diffuse oozing occurred due to disseminated intravascular coagulation. As a change in the concept, we decided to perform in situ liver splitting of the recipient liver to facilitate dissection of the retrohepatic IVC. Under hepatic inflow occlusion, right-left liver splitting was performed along the usual plane of extended left hepatectomy. The procedures time for recipient liver splitting and removal was 60 minutes. A modified right liver graft recovered from his daughter was implanted according to the standard procedures of LDLT. We think that recipient liver splitting is a feasible technical option for coping with difficult recipient hepatectomy, especially in patients with an enlarged stony-hard liver and heavy adhesion around the IVC.
\end{abstract}

Keywords: Hepatectomy; Massive bleeding; Coagulopathy; Transfusion; Liver splitting

\section{INTRODUCTION}

Enlarged stony-hard recipient livers are often observed in liver transplantation (LT) recipients, especially those with alcoholic liver disease. In such patients, recipient hepatectomy using the conventional surgical techniques is often demanding because of difficult dissection of the inferior vena cava (IVC) and excessive bleeding. A majority of surgeons majoring in LT have shared experience of hepatectomy for liver malignancies, thus they are accustomed to the anterior approach for hepatic resection in patients with huge hepatocellular carcinoma (HCC) located in the right liver $[1,2]$.
As a change in the concept, it is possible to apply such a liver splitting technique during recipient hepatectomy, like the anterior approach for $\mathrm{HCC}$ resection. The recipient liver is meant to be removed, thus there is no limitation on the duration of hepatic inflow occlusion [3,4]. Such type of liver splitting under prolonged hepatic inflow occlusion would facilitate the recipient hepatectomy than conventional dissection techniques when detachment of the retrohepatic IVC from the liver appears to be exceptionally difficult. We herein present our experience of the recipient liver splitting technique used for living donor liver transplantation (LDLT) in a patient with alcoholic liver cirrhosis. 


\section{HIGHLIGHTS}

- Recipient liver splitting under prolonged hepatic inflow occlusion facilitated the piggyback recipient hepatectomy in a patient with an enlarged stony-hard liver and heavy adhesion around the inferior vena cava.

\section{CASE REPORT}

This study was approved by the Institutional Review Board of Asan Medical Center (IRB No. IRB No. 2020-0836), which waived the requirement for informed consent.

A 48-year-old male patient diagnosed with alcoholic liver cirrhosis was admitted to our institution due to acuteon-chronic liver failure (Fig. 1). Blood tests showed total bilirubin level of $26.7 \mathrm{mg} / \mathrm{dL}$, creatinine level of $2.9 \mathrm{mg} /$ $\mathrm{dL}$, and prothrombin time-derived international normalized ratio of 1.75, thus making a Model for End-Stage Liver Disease (MELD) score of 35. This patient required inotropic agents to maintain his blood pressure, and thus he was cared for in the intensive care unit. This patient was enrolled into the Korean Network for Organ Sharing (KONOS) waitlist as the old KONOS category 2A. Because his condition had progressively deteriorated during waiting for priority allocation, we decided to perform LDLT.

The donor was the 21-year-old daughter of the patient. A modified right liver graft recovered from this donor weighed $760 \mathrm{~g}$, making a graft-to-recipient weight ratio of $0.89 \%$. The donor recovered uneventfully from the donor operation and was discharged at 9 days after the operation. During the recipient operation, we found that the native liver was stony-hard and heavily adherent to the retrohepatic IVC. During liver mobilization, diffuse oozing occurred from the dissected right liver surface and right subphrenic area, probably due to coagulopathy as a result of disseminated intravascular coagulation (DIC), which required more than 20 units of packed red blood cells (RBCs) until exposure of the right adrenal gland and partial dissection of the hepatoduodenal ligament. As a change in the concept, we decided to perform in situ liver splitting of the recipient liver to facilitate dissection of the retrohepatic IVC.

The hepatoduodenal ligament was dissected according to the standard procedures of LDLT using a right liver graft. The proximal bile duct was transected above the bifurcation level. Hepatic arteries and the main portal vein were isolated. A vascular tourniquet was applied to the distal part of the dissected common hepatic artery and the main portal vein to interrupt the hepatic inflow as in the usual Pringle maneuver. We searched for the easiest plane of parenchymal transection for liver splitting, by which we recognized that the usual right hepatectomy or extended left hepatectomy plane was most suitable for liver splitting. Under the hepatic inflow occlusion, right-left liver splitting was performed along the right side of the middle hepatic vein. After the ventral half of parenchymal transection was completed, the left hepatic artery and the left portal vein were transected as in the usual extended left hepatectomy (Fig. 2A and B). The Spigelian lobe was detached from the IVC through a right-to-left sided approach. The left liver along with the caudate lobe was removed (Fig. 2C) and the left-middle hepatic vein trunk was temporarily closed by sutures. Detachment of the right liver from the IVC was continued through a left-to-right side approach under prolonged hepatic inflow control. Finally, the right liver was removed from the retrohepatic IVC. Approximately 10 units

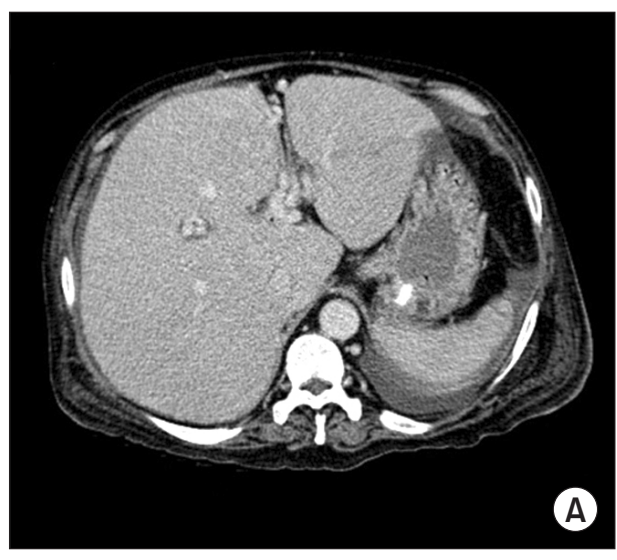

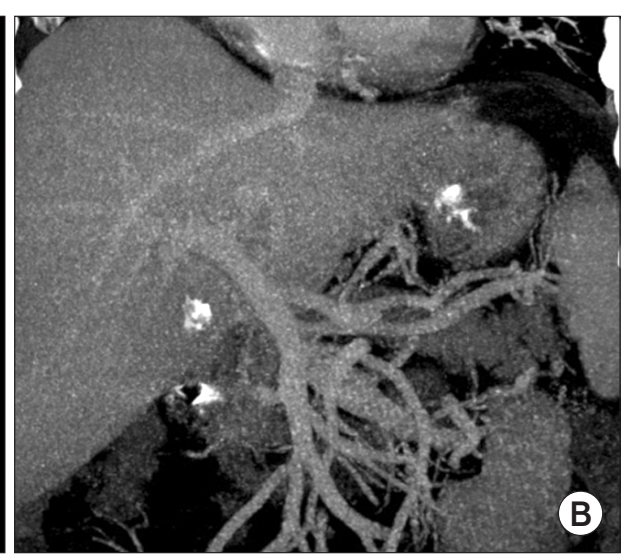

Fig. 1. Preoperative dynamic computed tomography findings. Hepatomegaly is visible (A) with poor development of portal vein collaterals $(\mathrm{B})$. 

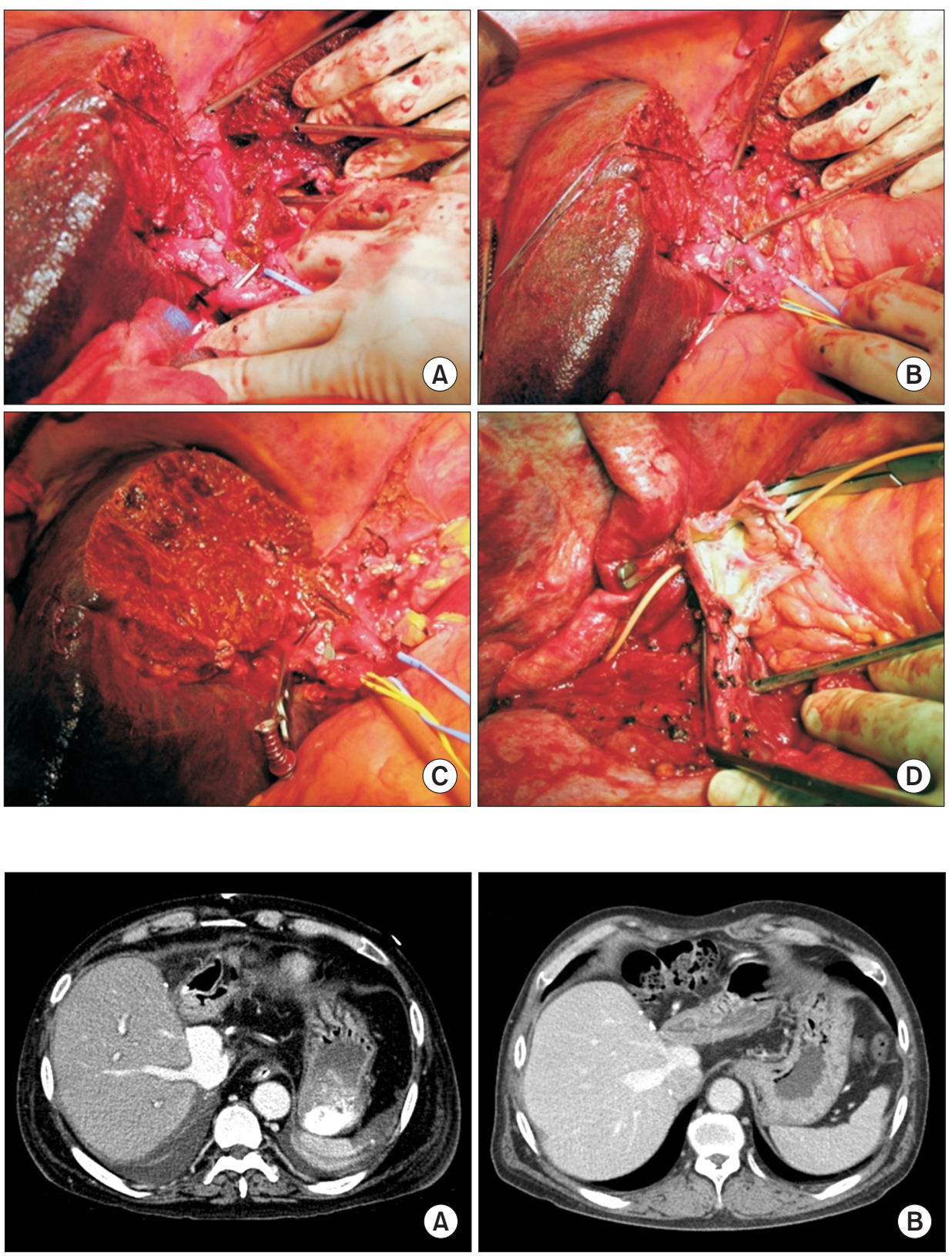

Fig. 2. Intraoperative photographs. (A, B) Right-left hemiliver splitting along the usual plane of extended left hepatectomy or right hepatectomy was performed under hepatic inflow occlusion. (C) The left liver along with the caudate lobe was removed with temporary closure of the left-middle hepatic vein trunk. (D) The three hepatic vein stumps in the recipient inferior vena cava were widely open, and the left-sided edge was reinforced with an autologous saphenous vein patch.

Fig. 3. Posttransplant dynamic computed tomography (CT) findings. (A) CT scan taken 2 weeks after transplantation shows no abnormal findings with bulged portion of the graft hepatic vein reconstruction. (B) CT scan taken 10 years after transplantation also shows no abnormal findings. of packed RBCs were infused during the 60-minute-long procedures of the recipient's liver splitting and removal. Approximately 20 minutes were needed for right-left hemihepatic parenchymal transection with Cavitron ultrasonic aspirator (CUSA). A total of 30 units of packed RBCs were infused during the whole recipient hepatectomy.

Soon after recipient hepatectomy, a modified right liver graft was implanted according to the standard procedures of LDLT. The three hepatic vein stumps in the recipient IVC were widely open under total clamping of the IVC, and the left-sided edge of the unified hepatic vein opening was reinforced with suturing of an autologous saphenous vein patch (Fig. 2D). The graft right and middle hepatic veins were unified at the back table for unification quilt venoplasty. The graft hepatic vein stump was anastomosed with the enlarged hepatic vein opening at the IVC. The recipient portal bifurcation was used for anastomosis with the graft portal vein. The single right hepatic artery was reconstructed with the corresponding recipient right hepatic artery under surgical microscopy. Biliary reconstruction 
was performed as duct-to-duct anastomosis.

The DIC state was not controlled all along the recipient operation, and diffuse oozing occurred after graft implantation. As a result, the total intraoperative requirement of blood components was 58 units of packed RBCs, 47 units of fresh frozen plasma, and 20 units of platelet concentrate. The total operation time from skin incision to skin closure was 14 hours 30 minutes because it took many hours to achieve complete bleeding control. The total portal vein occlusion time in combination with intermittent release was approximately 140 minutes, including in situ recipient liver splitting and graft implantation.

The pathology report of the explant liver showed that there was massive hepatic necrosis with fatty change and cholestasis, which was compatible with alcoholic fulminant liver failure. The patient recovered uneventfully from the LDLT operation (Fig. 3A). The general condition of the patient progressively improved, and finally the patient was discharged 50 days after LDLT. The patient has been doing well for 14 years after LDLT (Fig. 3B).

\section{DISCUSSION}

During the LT operation, excessive bleeding is one of the most serious problems. However, it occurs frequently due to impaired coagulation profiles and difficult hepatic dissection. We have performed more than 5,000 LDLT operations [5], in which a considerable proportion of recipients received high-volume transfusion due to massive intraoperative bleeding. The real-world situation of intraoperative bleeding during LDLT is different from that of deceased donor LT because the retrohepatic IVC needs to be completely preserved through a piggyback technique and all perihilar structures need to be meticulously dissected to preserve the small hepatic artery branches and hilar bile duct openings [6]. Massive bleeding and subsequent transfusion can cause various negative impacts on intraoperative management and posttransplant recovery [7]. Thus, it is highly recommended to minimize the intraoperative blood loss $[8,9]$.

The risk factors for intraoperative bleeding during the LT operation have been assessed in many studies. The MELD score is reported to be one of the important risk factors for massive transfusion [10]. Intraoperative cell salvage with autologous transfusion using a cell saver machine is effective in managing massive bleeding [11]. If liver cirrhosis is advanced with overt portal hypertension along poor development of portal collaterals, a considerable amount of bleeding inevitably occurs. Effective prevention of excessive bleeding is a matter of major concern during liver surgery including LT operation. Various surgical techniques, including the pinch-burn-cut techniques, high hilar dissection, and dissection with energy devices have been developed to reduce intraoperative bleeding during the LT operation $[12,13]$. We also reported the benefits of prolonged hepatic inflow occlusion during the LDLT operation $[3,4]$, in which prolonged inflow occlusion was effective in reducing bleeding during hepatic mobilization and dissection. However, prolonged prehepatic portal venous congestion or sinistral portal hypertension can be a potential risk factor for acute pancreatitis [14], thus irrelevant prolonged inflow occlusion is not recommended, especially in patients without portal vein collaterals.

The present case was performed in the era before the development of the current concept of prolonged hepatic inflow occlusion, but its background idea was identical with the current concepts. We have recently presented that occlusion of the hepatoduodenal ligament in LT recipients works as a stop-gap to weaken the bleeding-prone effect from portal hypertension [6,7]. If brisk bleeding occurs after damage of venous collaterals around the liver, it is usually difficult to achieve local bleeding control immediately. If venous collaterals exist proximal to the main portal vein, occlusion of the hepatoduodenal ligament will prevent bleeding. In patients with portal hypertension, there may be collaterals to compensate for portal hypertension. Such a situation may prevent potential inflow occlusion-induced splanchnic congestion because portal blood flow will bypass through the preexisting collaterals.

Recipient liver splitting under hepatic inflow control is not technically difficult because there is no need to preserve any part of the recipient liver. In the present case, right-left hemiliver splitting with CUSA took only 20 minutes, which is significantly shorter than the time period required for the usual right or left hepatectomy because such splitting is a type of destructive procedure. Once the recipient liver is separated with exposure of the retrohepatic IVC, recipient hepatectomy becomes easy because of the widely open operative field. If brisk bleeding occurs on the IVC surface, temporary interruption of the infrahepatic IVC with a vascular clamp is helpful during repair of the IVC defect. Therefore, recipient liver splitting appears to be a feasible option for facilitating recipient hepatectomy.

However, in real-world LT practice, recipient liver split- 
ting has been rarely performed probably because many surgeons think that recipient hepatectomy can be performed through other methods. One of the preferred methods is total hepatic vascular exclusion. It has not been frequently performed during hepatobiliary surgery [15], but it is a part of essential procedures for LT. Another method is application of portal flow bypass using active venovenous bypass, in which the hepatoduodenal ligament can be totally transected without the risk of splanchnic congestion and the IVC can be clamped for a prolonged period without risk of vital sign instability. Our recipient liver splitting technique cannot be applicable to patients with HCC, but these two methods can be useful regardless of HCC. Instead of the usual right-sided approach or recipient liver splitting, recipient hepatectomy through a left-sided approach under hepatoduodenal ligament occlusion can be used. This procedure facilitates dissection of the left liver and spigelian lobe from the retrohepatic IVC, thus the operation time and intraoperative bleeding can be reduced.

In conclusion, we think that recipient liver splitting is a feasible technical option for coping with difficult recipient hepatectomy, especially in patients with an enlarged stonyhard liver and heavy adhesion around the retrohepatic IVC.

\section{ACKNOWLEDGMENTS}

\section{Conflict of Interest}

No potential conflict of interest relevant to this article was reported.

\section{Funding/Support}

This study was supported by research grant from the Korean Society for Transplantation (2021-00-03004-016).

\section{ORCID}

Sung-Min Kim

Shin Hwang

Deok-Bog Moon

Dong-Hwan Jung

https://orcid.org/0000-0003-2079-6141 https://orcid.org/0000-0002-9045-2531 https://orcid.org/0000-0002-8209-3540 https://orcid.org/0000-0001-5984-023X

\section{Author Contributions}

Conceptualization: SH. Data curation: SMK, SH. Formal analysis: SH. Methodology: DBM, DHJ. Project administration: SH. Visualization: SH. Writing-original draft: SMK, SH. Writing-review \& editing: all authors.

\section{REFERENCES}

1. Hwang S, Moon DB, Lee SG. Liver transplantation and conventional surgery for advanced hepatocellular carcinoma. Transpl Int 2010;23:723-7.

2. Jabir MA, Hamza HM, Fakhry H, Amira G, Hatano E, Uemoto S. Anterior versus conventional approach for resection of large right lobe hepatocellular carcinoma. J Gastrointest Cancer 2017;48:25-30.

3. Choi JU, Hwang S, Chung IJ, Kang SH, Ahn CS, Moon $\mathrm{DB}$, et al. Prolonged hepatic inflow occlusion to reduce bleeding during recipient hepatectomy in living donor liver transplantation. Korean J Transplant 2020;34:5561.

4. Choi JU, Hwang S, Ahn CS, Moon DB, Ha TY, Kim KH, et al. Prolonged occlusion of the hepatoduodenal ligament to reduce risk of bleeding and tumor spread during recipient hepatectomy for living donor liver transplantation. Ann Hepatobiliary Pancreat Surg 2019;23:61-4.

5. Lee SG, Song GW, Yoon YI. An exceptional series: 5000 living donor liver transplantations at Asan Medical Center, Seoul, Korea. Transplantation 2019;103:173941.

6. Hwang S, Lee SG, Lee YJ, Sung KB, Park KM, Kim KH, et al. Lessons learned from 1,000 living donor liver transplantations in a single center: how to make living donations safe. Liver Transpl 2006;12:920-7.

7. Pustavoitau A, Lesley M, Ariyo P, Latif A, Villamayor AJ, Frank SM, et al. Predictive modeling of massive transfusion requirements during liver transplantation and its potential to reduce utilization of blood bank resources. Anesth Analg 2017;124:1644-52.

8. Donohue $\mathrm{Cl}$, Mallett SV. Reducing transfusion requirements in liver transplantation. World J Transplant 2015;5:165-82.

9. Steib A, Freys G, Lehmann C, Meyer C, Mahoudeau G. Intraoperative blood losses and transfusion requirements during adult liver transplantation remain difficult to predict. Can J Anaesth 2001;48:1075-9.

10. Massicotte L, Beaulieu D, Roy JD, Marleau D, Vandenbroucke $\mathrm{F}$, Dagenais $\mathrm{M}$, et al. MELD score and blood product requirements during liver transplantation: no link. Transplantation 2009;87:1689-94.

11. Pinto MA, Chedid MF, Sekine L, Schmidt AP, Capra RP, Prediger $C$, et al. Intraoperative cell salvage with autologous transfusion in liver transplantation. World $\mathrm{J}$ Gastrointest Surg 2019;11:11-8. 
12. Park YK, Kim BW, Wang HJ, Xu W. Usefulness of the Pinch-Burn-Cut (PBC) technique for recipient hepatectomy in liver transplantation. Korean J Hepatobiliary Pancreat Surg 2012;16:13-6.

13. Lee KW, Joh JW, Kim SJ, Choi SH, Heo JS, Lee HH, et al. High hilar dissection: new technique to reduce biliary complication in living donor liver transplantation. Liver Transpl 2004;10:1158-62.
14. Verran DJ, Gurkan A, Chui AK, Dilworth P, Koorey D, McCaughan $\mathrm{G}$, et al. Pancreatitis in adult orthotopic liver allograft recipients: risk factors and outcome. Liver Transpl 2000;6:362-6.

15. Smyrniotis VE, Kostopanagiotou GG, Gamaletsos EL, Vassiliou JG, Voros DC, Fotopoulos AC, et al. Total versus selective hepatic vascular exclusion in major liver resections. Am J Surg 2002;183:173-8. 